\title{
Analysis of Electrocardiographic Changes in Cerebrovascular Accidents
}

\author{
Dr. Sharat Kumar B Jaikar ${ }^{1}$, Dr. Divya NS ${ }^{2}$, Dr. Rajan $\mathrm{C}^{3}$ \\ ${ }^{1}$ (Department Of Medicine, DM-WIMS Medical College, Wayanadu, Kerela) \\ ${ }^{2}$ (Department Of Pathology, DM-WIMS MedicalCollege, Wayanadu, Kerela) \\ ${ }^{3}$ (Department Of Medicine, DM-WIMS Medical College,Wayanadu, Kerela)
}

\begin{abstract}
Background- the interrelationship of cerebrovascular disease and cardiovascular disease has been repeatedly emphasised and the decompensation in one system can adversely affect the other. The possibility of cerebrovascular accidents (CVA) could result in cardiac rhythm disturbances or structural damage and may be associated with abnormal Electro-Cardiographic Changes (ECG) even in patients without apparent heart disease.

Methods- a total of 60 CVA cases have been evaluated and associated ECG changes were analyzed. Results- in the present study, $83.3 \%$ patients showed abnormal ECG changes. All patients (100\%) with cerebral haemorrhage, Sub-arachanoid Haemorrhage (SAH), Cortical Venous Thrombosis (CVT) had ECG changes whereas it was only $64.28 \%$ in Cerebral Thrombosis.

Conclusion- different ECG changes are observed in CVA cases even in absence of primary cardiac disease and these ECG changes can be predictor of prognosis.
\end{abstract}

Keywords: cerebrovascular accidents, cerebral hemorrhage, ECG, prognosis, stroke.

\section{Introduction}

The term cerebrovascular accident or stroke includes any neurological dysfunction of sudden onset secondary to interruption in blood flow or by hemorrhage in to or around brain other than trauma. It is the leading cause of mortality and morbidity worldwide and accounts for $20 \%$ of all neurological admissions in India. Role of heart as a cause of stroke has received much attention in recent past years and the decompensation in either central nervous system or cardiovascular system may adversely affect each other, whether or not the patient has recognized disease of both systems ${ }^{[1]}$. Numerous studies have demonstrated the fact that primary neurologic abnormalities may produce ECG changes without any myocardial lesion. ECG changes affecting $\mathrm{T}$ wave, $\mathrm{U}$ wave, S-T segment, Q-T interval and arrhythmias have been reported. These changes may resemble those of myocardial ischemia and acute myocardial infarction, leading to misinterpretation and delay in operative management of sub-arachanoid haemorrhage ${ }^{[2]}$. There are evidences suggesting, that patients who had ECG changes following cerebrovascular accidents had poor prognosis compared to those who did not show any ECG changes. Approximately 2-6\% of all stroke patients die from cardiac causes in first three months after ischemic stroke ${ }^{[3]}$.

In the view of above speculations, this study was undertaken to identify the Electrocardiographic changes produced due to cerebrovascular accidents.

\section{Methodology}

This prospective study was carried out in a tertiary care hospital, during 2007- 2010. 60 cases consecutively admitted with acute cerebrovascular accidents with ECG changes were included in the study group. A detailed clinical history and thorough physical examination with emphasis to nervous system and cardiovascular system was done as per the standard proforma.

Aims of the study-

- to study the incidence of ECG changes in patients with acute stroke

- to study the nature and types of ECG changes in different types of strokes

- to find out whether there is any prognostic significance for these abnormal ECG findings in patients with acute stroke. changes.

Inclusion criteria- cases of 'stroke syndrome' and admitted within 72 hours and patients having ECG

Exclusion criteria- Stroke cases which came after 72 hours, the individuals with head injury and known cardiac, hepatic and renal diseases.

12 Lead Electrocardiogram was taken for all the cases within 24 hours of admission and subsequently repeated on $3^{\text {rd }}$ day, $7^{\text {th }}$ day and $30^{\text {th }}$ day for follow up. CT scan brain was taken and all patients were subjected 
to investigations like Trop-i or Trop-t, $2 \mathrm{~d}$ echocardiogram, serum electrolytes, urine analysis, blood sugar, blood urea, lipid profile and relevant serological tests.

The diagnosis of CVA was made by recognizing 'stroke syndrome' by following criteria ${ }^{[4]}$

- temporal profile of the clinical syndrome

- evidence of focal brain damage/disease

- clinical setting

Various types of cerebrovascular accidents like Cerebral Thrombosis, Cortical Venous Thrombosis, Cerebral Embolism, Intracranial Hemorrhage and Subarachanoid Hemorrhage were observed and documented (fig 4). A detailed analysis and interpretation of ECG changes like sinus arrhythmia, sinus bradycardia, sinus tachycardia, abnormal $\mathrm{Q}$ wave, $\mathrm{U}$ wave, $\mathrm{T}$ wave, right and left ventricular hypertrophy, $\mathrm{S}$-T segment elevation/ depression, $\mathrm{T}$ wave inversion and prolongation of Q-T interval was done.

During the hospital stay all cases were followed up regularly with repeat ECG's. Patients were managed with general and specific measures according to the type of stroke. The only prognostic criterion considered was the mortality among patients within study period.

\section{Results}

60 patients admitted with acute stroke were studied in detail and following observations were noted:

Age and sex distribution- out of 60 total cases, 36 were males and 24 were females (3:2). Strokes are common in middle age and elderly patients and incidence being $76.6 \%$ in the age group above 40 years. Cerebral thrombosis and intracerebral haemorrhage is more common in males, whereas CVT is exclusively confined to females.

Types of stroke- CT scan was used to identify the type of stroke. Table 1 shows the incidence of different types of stroke. Cerebral thrombosis contributed highest number of cases i.e., $46.6 \%$ followed by Intracerebral hemorrhage (40\%) and CVT (6.66\%). No cases of cerebral embolism were encountered. Out of 60 cases, 50 showed ECG abnormalities (83.3\%). Remaining 10 cases (16.6\%) did not show any changes in ECG. Fig/graph-1 shows different type of ECG changes encountered. T wave inversion (36\%) and S-T segment depression (20\%) was commonly noted. Table 2 highlights the specific ECG changes in different types of stroke.

18 patients out of 60 expired after $30^{\text {th }}$ day following CVA. Mortality was taken as the prognostic indicator. Out of 18 expired cases, 16 had ECG changes and 2 patients did not have any ECG changes (Fig/ graph 2). It was also noted that patients with ECG changes had bad prognosis (mortality $32 \%$ ) as compared to patients without ECG changes (mortality 20\%).

\section{Discussion}

A close relationship between cerebrovascular accidents and cardiovascular diseases is a well known fact. Cardiovascular diseases may be coexisting or a causative condition for stroke. Numerous studies have documented that the primary neurologic abnormalities like CVA can produce ECG changes like affecting S-T segment, $\mathrm{T}$ wave, $\mathrm{U}$ wave and Q-T interval. A high incidence of cardiac arrhythmias have also been reported in acute cerebrovascular lesions ${ }^{[5,6,7,8]}$. In clinical practice, ECG changes may be misinterpreted as the changes of myocardial ischemia thereby affecting the assessment of immediate prognosis and thus delaying the definite surgical management in these stroke patients.

Cerebral infarction and Intracerebral hemorrhages are more common in males, which was similar to our study and incidence of cerebral embolism varies from $18-23 \%{ }^{[9]}$. No cases of cerebral embolism were encountered in our study. As our study group was small, studies with large number of patients may be required to demonstrate the actual incidence of cerebral embolism. Maximum stroke cases were seen between 60-90 years because of strict control of hypertension and other risk factors, incidence of stroke is less in middle age group.

According to National Institute of Neurological Diseases and Stroke ${ }^{[10]}$, the incidence of Intracerebral hemorrhage is $40 \%$, whereas in our study it is only $13 \%$. Hemorrhagic strokes are more common in Indians compared to Western population. CVT was more common in our study. In various control studies, a comparison of ECG changes and arrhythmias seen in stroke patients, were made with the ECG finding of age and sex matched controls. The differences in the ECG findings were statistically significant and these ECG changes were attributed to cerebral cause. In the present study, $83.3 \%$ of stoke cases showed abnormal ECG changes, which was similar to the study conducted by Mathur et al ${ }^{[11]}$. We observed that all the cases of Intracerebral and Subarachanoid hemorrhages showed ECG changes. 64.28\% cases of Cerebral Thrombosis and 4 cases of CVT was associated abnormal ECG.

$\mathrm{S}-\mathrm{T}$ segment depression and $\mathrm{T}$ wave inversion were among the commonest changes observed in stroke. $\mathrm{S}$-T changes were seen in all cases of stroke with or without associated cardiac disease (Fig 3 ) and were frequently seen in ischemic strokes rather than hemorrhagic strokes. Increased sympathetic has been proposed as the cause for these ST-T changes and these changes persisted till the end of follow up ${ }^{[12]}$. Arrhythmias are likely 
to contribute to the sudden unexpected mortality following stroke, therefore patients require continuous cardiac monitoring ${ }^{[13]}$. In our study, all the 4 cases of Atrial Fibrillation became normal during the follow up. Other ECG changes like Q-Tc prolongation, sinus tachycardia, sinus bradycardia and prominent U waves were also encountered similar to other studies quoted in the literature ${ }^{[11,12]}$.

Few ECG changes are specific to particular type of stroke ${ }^{[14]}$. In Cerebral hemorrhages, all types of ECG changes were observed i.e., ST-T changes, Q-Tc prolongation, arrhythmias, sinus tachycardia except for sinus bradycardia and prominent $\mathrm{U}$ waves and mortality was highest among the patients with ST-T changes. 4 cases of CVT had sinus bradycardia and T wave inversion.

An attempt was made to find out the prognostic importance of ECG changes in stroke patients [Fig/graph 2]. The patients who had persistent ECG changes had bad prognosis compared to patients with normal ECG which was similar to the observations made by Bozluoclay et al ${ }^{[5]}$, and Dogan et al ${ }^{[15]}$. Our study concluded that ST-T segment changes, prolonged Q-Tc and abnormal U waves were univariate predictors of mortality.

\section{Figures And Tables}

\begin{tabular}{|lll|}
\hline Type of stroke & No. Of patients & Percentage \% \\
& & \\
\hline Cerebral Haemorrhage & 24 & 40 \\
\hline Subarachanoid Haemorrhage & 04 & 6.6 \\
\hline Cerebral Thombosis & 28 & 46.6 \\
\hline Cortical Venous Thombosis & 04 & 6.6 \\
\hline TOTAL & $\mathbf{6 0}$ & $\mathbf{1 0 0}$ \\
\hline
\end{tabular}

TABLE 1: Incidence of different types of stroke

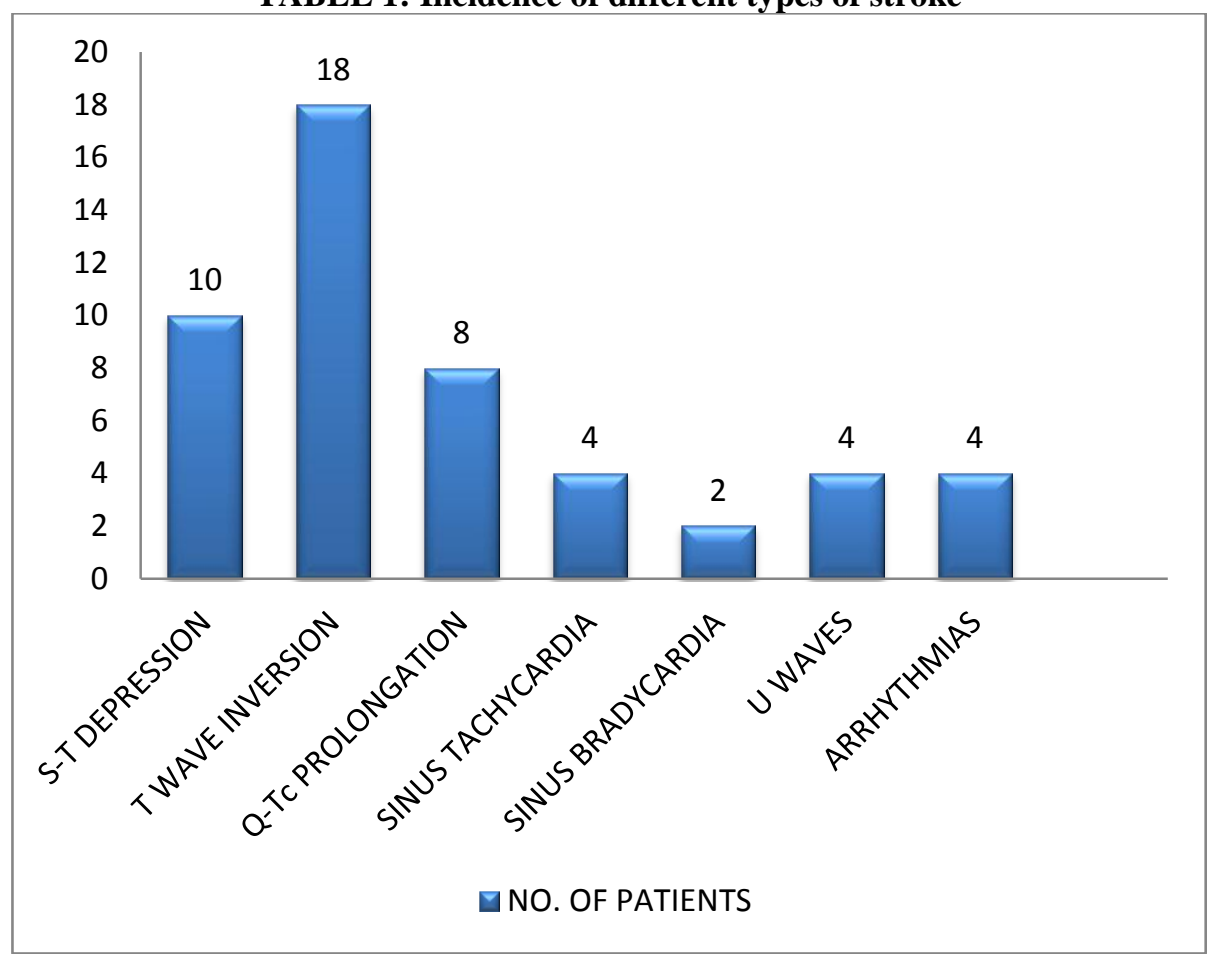

FIG 1: Graph showing different types of ECG changes in CVA

\begin{tabular}{|c|c|c|c|c|c|}
\hline $\begin{array}{l}\text { Types Of ECG } \\
\text { Changes }\end{array}$ & Cerebral Thombosis & $\begin{array}{l}\text { Cerebral } \\
\text { Haemorrhage }\end{array}$ & $\begin{array}{l}\text { Subarachanoid } \\
\text { Haemorrhage }\end{array}$ & $\begin{array}{l}\text { Cortical Venous } \\
\text { Thombosis }\end{array}$ & TOTAL \\
\hline $\begin{array}{l}\text { S-T segment } \\
\text { abnormalities }\end{array}$ & 2 & 6 & 2 & - & 10 \\
\hline $\begin{array}{l}\text { T wave } \\
\text { abnormalities }\end{array}$ & 14 & 2 & - & 2 & 18 \\
\hline Q-Tc prolongation & - & 8 & - & - & 8 \\
\hline Sinus tachycardia & 2 & 2 & - & - & 4 \\
\hline Sinus bradycardia & - & - & - & 2 & 2 \\
\hline U waves & - & 2 & 2 & - & 4 \\
\hline Arrhythmias & - & 4 & - & - & 4 \\
\hline
\end{tabular}


TABLE 2: Types of ECG changes in different types of stroke

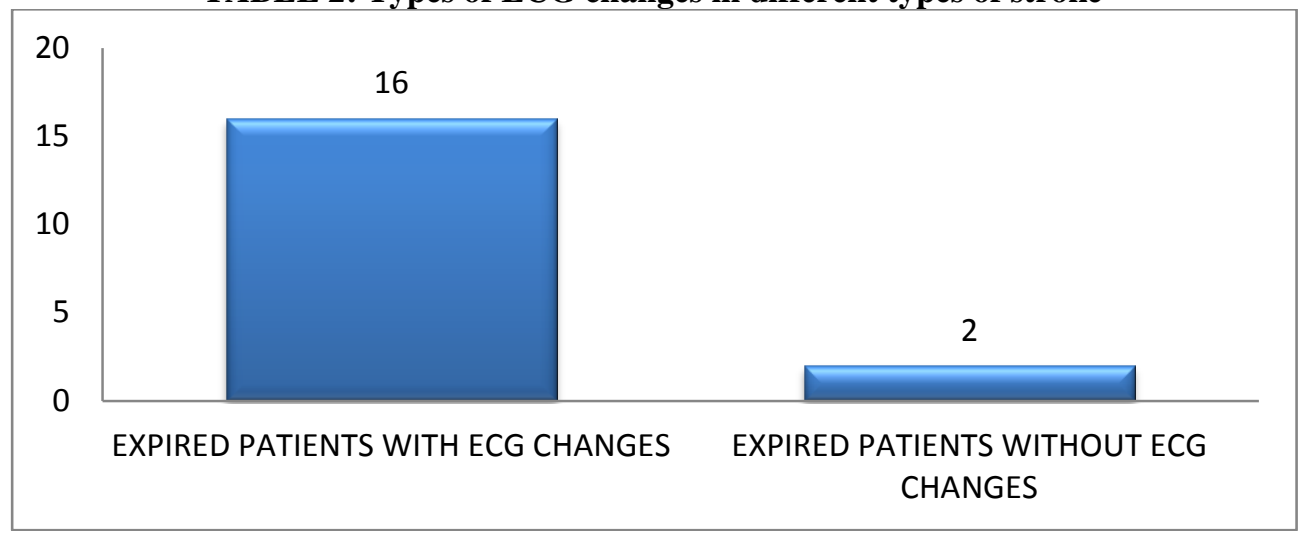

FIG 2: Graph showing Mortality rate in CVA patients

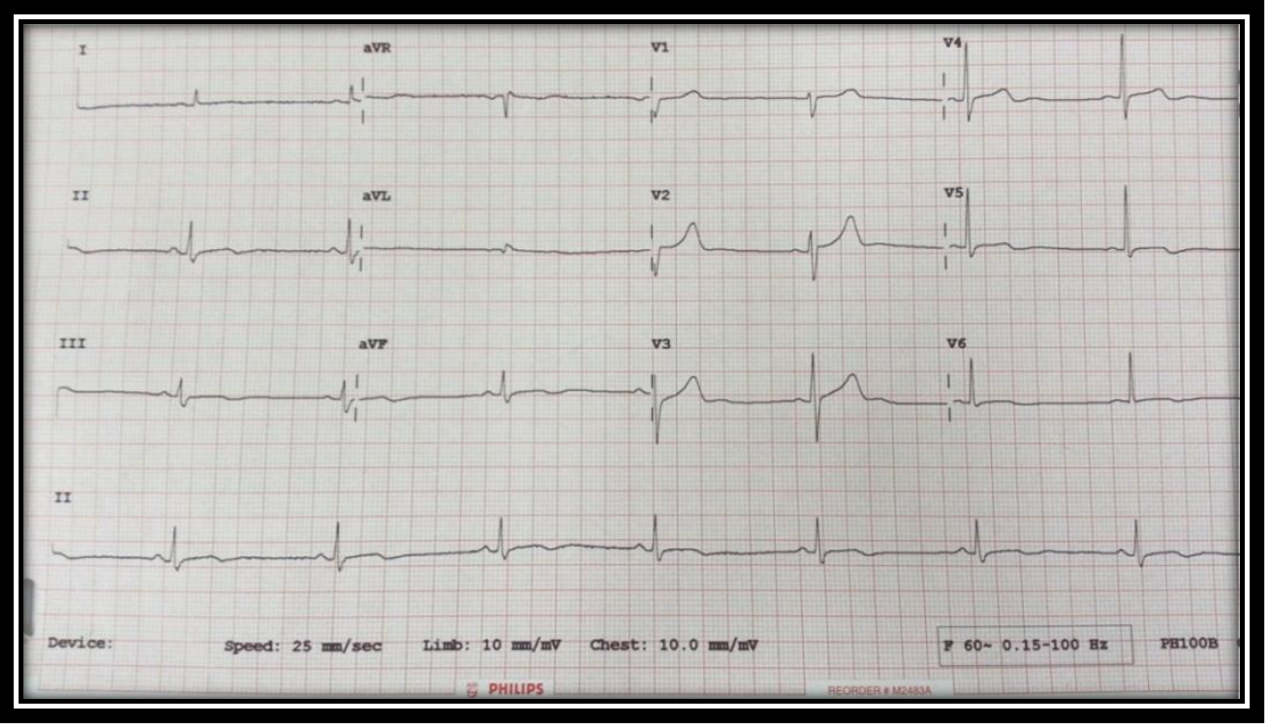

FIG 3: ECG of a stroke patient (with cardiac disease) showing bradycardia with ST-T abnormality in inferior and lateral leads

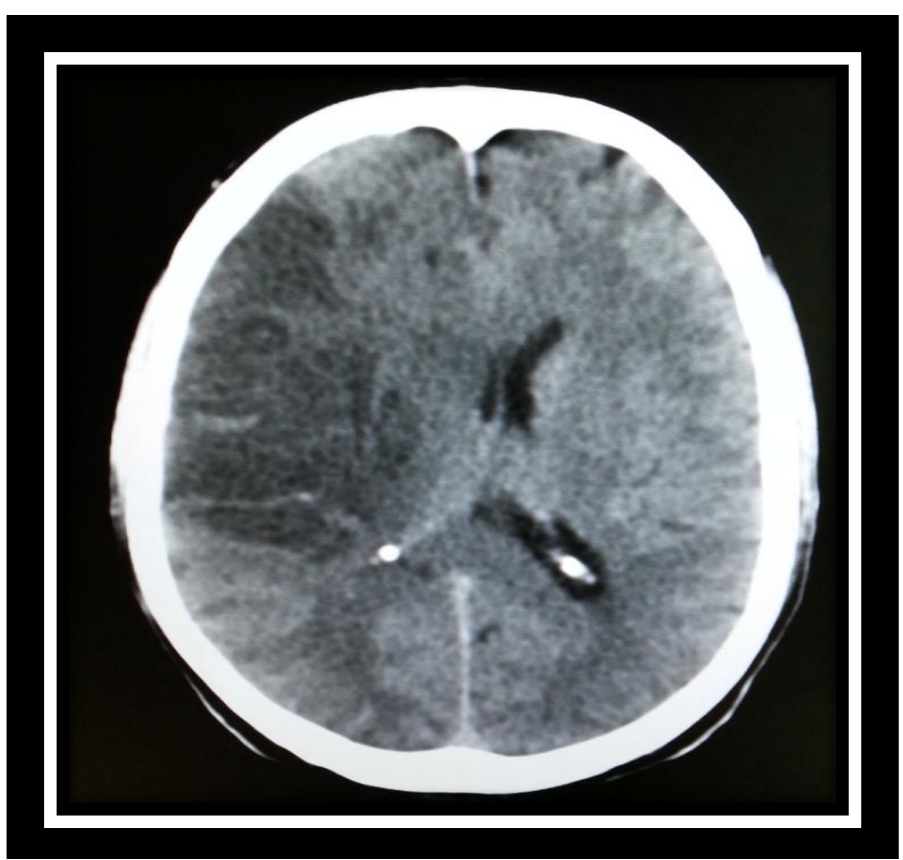

FIG 4: CT scan showing massive right MCA infarct with perilesional oedema and midline shift 


\section{Conclusions}

This study confirms the role of ECGs as a part of routine investigations of patients admitted with stroke. Knowledge of these ECG changes will prevent unnecessary delay in proper assessment and operative management of stroke patients. Mortality is high among the patients who had persistent ECG changes, therefore it is important to monitor cardiac activity in all the patients of stroke.

\section{References}

[1]. Dimant J, Grob D, Electrocardiographic changes and myocardial damage in patients with acute cerebrovascular accidents, Stroke, $8,1977,455$

[2]. Croopp GJ, Manning GW, Electrocardiographic changes stimulating myocardial ischemia and interactions associated with spontaneous intracranial haemorrhage, Circulation, 22, 1960, 25-38.

[3]. Prosser J, Mac Gregor L, R Kennedy, D Hans- Christoph, Predictors of early cardiac morbidity and mortality after ischemic stroke, Stroke, 38, 2007, 2295-2302.

[4]. Adams RD, Victor M, Ropper AH, Cerebrovascular diseases in principles of Neurology, USA, Me Grow Hill Health Professions Divisions, $8^{\text {th }}$ Ed, 2005,660- 746 .

[5]. Bozluolcay M, Ince B, Celik Y, Harmanchi H, Electrocardiographic findings and prognosis in ischemic stroke, Neurology India J, 51(4), 2003,500-02.

[6]. Byer E, Ashman R, Toth LA, Electrocardiograms with large upright T waves and long Q-T intervals, Am Heart J, 33,1947, 796- 99.

[7]. Fentz V, Gormsen J, Electrocardiographic patterns in patients with cerebrovascular accidents, Circulation, 25, 1962, 22-28.

[8]. Stern S, Lavy S, Carmon A, Electrocardiographic patterns in Haemorrhagic Stroke, J Neurol Science,8,1968, 61-67.

[9]. Wolf PA, Kanel WB, D'gastino RB, Epidemiology of stroke, 59,Cerebrovascular disease- pathophysiology, diagnosis and management, Guisbug MD, USA- Blackwell Science Ltd, 2, 1998, 834-49.

[10]. Foulkes MA, The stroke data bank- desing, methods and base line characteristics, Stroke, 19, 1988, $547-554$.

[11]. Mathur KS, Wahal PK, Singhal RK, Electrocardiographic abnormalities associated with cerebrovasular accidents. J of Indian Medical Association, 46(11), 1966, 599-601.

[12]. Goldstein DS, The electrocardiogram in stroke- Relationship to pathophysiological type and comparison with prior tracings, Stroke, 10, 1979, 253- 59.

[13]. Oppenheimer SM, Hachinski VC, The cardiac consequences of stroke, Neurologic Clin J, 10(1), 1992, $167-76$.

[14]. Mehtha PJ, Desai IN, 6 Electrocardiographic diagnosis, J of Applied Med, 12(10), 1986, 683-84.

[15]. Dogan A, Tunc E, Ozturk M, Kerman M, Electrocardiographic changes in patients with ischemic stroke and their prognostic importance, Int J Clin Practice, 58(5), 2004, 436- 440. 